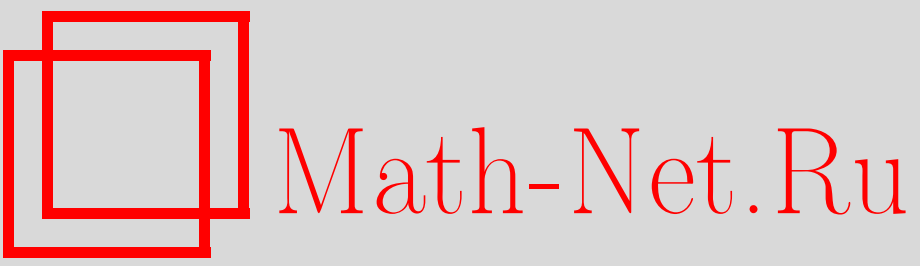

В. Е. Шестопал, Многопараметрические модели случайных блужданий в решеточных неупорядоченных системах, ТМФ, 1999, том 119, номер 2, 332-344

DOI: https://doi.org/10.4213/tmf742

Использование Общероссийского математического портала Math-Net.Ru подразумевает, что вы прочитали и согласны с пользовательским соглашением

http://www.mathnet.ru/rus/agreement

Параметры загрузки:

IP: 3.85 .73 .92

26 апреля 2023 г., 03:10:01 


\title{
МНОГОПАРАМЕТРИЧЕСКИЕ МОДЕЛИ СЛУЧАЙНЫХ БЛУЖДАНИЙ В РЕШЕТОЧНЫХ НЕУПОРЯДОЧЕННЫХ СИСТЕМАХ
}

\begin{abstract}
Предлагаются многомерные асимптотически точно решаемые модели случайных блужданий в случайной стационарной решеточной среде. Эти модели отличаются от известных сколь угодно большим числом независимых локальных случайных параметров, приходящихся на один узел решетки, и допускают медленное убывание интенсивностей с увеличением расстояний между узлами. Предложенные модели описывают, в частности, первые нетривиальные точно решаемые многомерные системы с симметричным взаимодействием.
\end{abstract}

1. Изучение случайных блужданий в неупорядоченных решеточных системах (СБНРС) имеет большое значение для различных областей естествознания и связано с проблемами статистической физики и теории случайных процессов (см. недавние работы [1-12]).

С точки зрения многих приложений основная задача теории СБНРС - получение усредненных по случайности среды решений системы кинетических уравнений, т.е. прямых уравнений Колмогорова для необрываюшейся цепи Маркова

$$
\frac{d \widetilde{P}_{x y}}{d t}=-\sum_{z}\left(\widetilde{w}_{z x} \widetilde{P}_{x y}-\widetilde{w}_{x z} \widetilde{P}_{z y}\right), \quad \widetilde{P}_{x y}(t=0)=\delta_{x y}
$$

где $x, y, z$ пробегают целочисленную решетку $Z^{d} ; \widetilde{P}_{x y}(t)$ - вероятность найти блуждающий объект в момент $t$ в узле $x$, если сначала он находился в $y$; величины $\widetilde{w}_{x z}$ (интенсивности переходов (или скорости прыжков) из $z$ в $x$ ) - это неотрицательные случайные величины, по которым ведется усреднение. Результат усреднения величины $f$ обозначаем далее $E(f)$ или $\prec f \succ$. Особое значение для приложений имеет требование стохастической однородности или инвариантности распределения случайных локальных параметров среды блужданий относительно сдвигов решетки или сдвигов на векторы из подрешетки максимального ранга (так называемая "инвариантность в среднем"). Такая "неполная" инвариантность имеет место в реальных задачах физики решеточных

${ }^{*}$ Институт теоретической и эксперементальной физики, Москва, Россия. E-mail: shestopal@vitep5.itep.ru 
систем с конечной элементарной ячейкой (фундаментальной областью). Ее учет важен также для изучения возможных свойств блужданий в сложных аморфных средах, хорошо описываемых в терминах непрерывного предела неупорядоченных решеточных систем.

Сложность изучения проблемы блужданий в неупорядоченных системах видна из следующего. В настоящее время среди известных нетривиальных асимптотически (при $t \rightarrow+\infty$ ) точно решаемых моделей СБНРС (см. [1-3, 10, 11] и обзор [4]) важным свойством многих реальных процессов - симметричностью ("обратимостью в пространстве") скоростей прыжков между узлами (когда $\widetilde{w}_{x z}=\widetilde{w}_{z x}$ ) - обладают лишь одномерные модели с конечным радиусом взаимодействия $[1,4,11]$. Отметим, что известными из литературы нетривиальными асимптотически точно решаемыми моделями СБНРС c дальними прыжками и случайными, флуктуируюшими до нуля на всех расстояниях интенсивностями являются несимметричная модель [10], обобшаюшая "модель изотропных случайных прыжков" (первоначально с переносом лишш в ближайшие узлы решетки $[3,4])$ и модели с принудительным сносом в конусе направлений и сводящиеся к ним [10]. В ряде случаев с более обшей локальной случайностью и быстрым спаданием интенсивностей известны лишь качественные результаты и оценки асимптотических характеристик $[4,7]$.

Цель предлагаемой работы - последовательное, конструктивное описание значительно более широких классов асимптотически точно решаемых инвариантных в среднем моделей СБНРС на решетке, обладаюших стационарной локальной случайностью скоростей прыжков и большим числом приходяшихся на один узел случайных параметров. Совершенно новыми среди них являются модели многомерных процессов с симметричным (обратимым в пространстве узлов-состояний цепи) переносом. При этом допускается медленное убывание интенсивностей переходов при увеличении расстояний между узлами. Получение долговременных асимптотических разложений основано на развитии методов работ $[10,11]$. Приведенной в [10] аналитической техники также вполне достаточно, чтобы проверять условия включения многих интересных моделей в предлагаемые здесь новые классы асимптотически точно решаемых систем и проводить в них полный расчет асимптотических разложений.

В настояшей работе строятся и подробно исследуются асимптотические решения для первого из классов асимптотически точно решаемых моделей, приводятся примеры систем из этого класса, обладаюших указанными выше новыми свойствами. Кроме того, описывается, каким образом можно строить и исследовать значительно более общие, чем первый, классы моделей блужданий в неупорядоченных средах. Отмечается также, что многие основные результаты данной работы могут быть правильно получены недостаточно математически обоснованным методом, недавно положенным в основу детального исследования долговременной асимптотики ряда реальных процессов [13].

Мы будем опираться на известную связь между соответствуюшими асимптотиками больших времен и малых значений параметра Лапласа, т.е. $\prec \widetilde{P}_{x y}(t \rightarrow+\infty) \succ$ и $\prec \widetilde{P}_{x y}(\lambda \rightarrow 0) \succ, \operatorname{Re}(\lambda) \geqslant 0$ (тауберовы и абелевы теоремы), обозначая переход к преоб- 
разованию Лапласа переименованием переменной:

$$
f(t) \rightarrow f(\lambda)=\int_{0}^{+\infty} e^{-t \lambda} f(t) d t
$$

2. Первый из изучаемых в работе класс асимптотически точно решаемых цепей Маркова (1) описывается с помощью систем уравнений

$$
\frac{d \widetilde{P}_{x y}^{m n}}{d t}=-\sum_{z} \sum_{i, j, l=1}^{N} \alpha_{x}^{m i} A_{x-z}^{i j} \beta_{z}^{j l} \widetilde{P}_{z y}^{l n}, \quad \widetilde{P}_{x y}^{m n}(t=0)=\delta_{x y} \delta_{m n}
$$

где индексы $x, y, z$ пробегают множество узлов решетки $Z^{d}$, образованной фиксированным типом узлов в элементарных ячейках, индексы $i, j, l, m, n=1, \ldots, N$ нумеруют типы узлов в пределах ячейки.

Относительно остальных вешественных параметров системы предполагается, что выполняются следующие условия.

Условие 1. При различных $x$ пары числовых $(N \times N)$-матриц $\left(\alpha_{x}^{i j}, \beta_{x}^{i j}\right)$ - независимые одинаково распределенные случайные величины, и сушествует достаточно много обратных матричных моментов вида $\prec\left(\alpha_{x} \beta_{x}\right)^{-r} \succ, \prec\left(\beta_{x} \alpha_{x}\right)^{-r} \succ, \prec\left(\alpha_{x} \beta_{x}\right)^{-r} \beta_{x}^{-1} \succ$, $\prec\left(\beta_{x} \alpha_{x}\right)^{-r} \alpha_{x}^{-1} \succ$, где $r \geqslant 1,\left(\alpha_{x}\right)^{m n}=\alpha_{x}^{m n},(\alpha)_{x y}^{m n}=\alpha_{x}^{m n} \delta_{x y}$, аналогично для $\beta$.

Условие 2. Оператор

$$
p_{x}^{m} \rightarrow-(A p)_{x}^{m}=-\sum_{z} \sum_{i=1}^{N} A_{x-z}^{m i} p_{z}^{i} \quad \text { при } \quad \lambda \rightarrow 0, \quad \operatorname{Re}(\lambda) \geqslant 0
$$

имеет резольвенту, которая равномерно по $k \in[-\pi, \pi]$ удовлетворяет оценке

$$
\left|\sum_{z} e^{-i k z}\left(\left(\prec \frac{\lambda}{\beta \alpha} \succ+A\right)^{-1}\right)_{z 0}^{m n}\right|=O\left(\left|\lambda^{-r_{1}}\right|\right) .
$$

Условие 3. Тот же оператор $А$ удовлетворяет оценке

$$
\int_{-\pi}^{\pi} d k\left|\sum_{z} e^{-i k z}\left(\left(\prec \frac{\lambda}{\beta \alpha} \succ+A\right)^{-1}\right)_{z 0}^{m n}\right|=O\left(\left|\lambda^{-r_{2}}\right|\right),
$$

причем $r_{1}+r_{2}<2, r_{2}<1$.

Выполняется также хотя бы одно из следующих четырех условий:

Условие 4. При конечных величинах $E\left(\alpha_{x}\right), E\left(\alpha_{x}^{-1}\right), E\left(\beta_{x} \beta_{x}^{+}\right)$имеем

$$
\left|\left(\frac{1}{\bar{\lambda}+\beta^{+} A^{+} \alpha^{+}} \times \frac{1}{\lambda+\alpha A \beta}\right)_{z z}^{m m}\right|=O\left(|\lambda|^{-r_{3}}\right),
$$

где $\gamma^{+}$есть эрмитово сопряженный к $\gamma$ оператор. 
Условие 5. При конечных $E\left(\beta_{x}\right), E\left(\beta_{x}^{-1}\right), E\left(\alpha_{x}^{+} \alpha_{x}\right)$ имеем

$$
\left|\left(\frac{1}{\lambda+\alpha A \beta} \times \frac{1}{\bar{\lambda}+\beta^{+} A^{+} \alpha^{+}}\right)_{z z}^{m m}\right|=O\left(|\lambda|^{-r_{3}}\right) .
$$

Условие 6. При конечных $E\left(\alpha_{x}^{-1}\right), E\left(\beta_{x}^{-1}\right), E\left(\beta_{x} \beta_{x}^{+}\right)$верно соотношение (3).

Условие 7. При конечных $E\left(\alpha_{x}^{-1}\right), E\left(\beta_{x}^{-1}\right), E\left(\alpha_{x}^{+} \alpha_{x}\right)$ верно соотношение (4).

Основная интересуюшая нас проблема - нахождение асимптотики решения системы (2) при $t \rightarrow+\infty$ (или малых $|\lambda|)$, усредненного по наборам $\left\{\left(\alpha_{x}, \beta_{x}\right) \mid x \in Z^{d}\right\}$, т.е. величины

$$
P_{x y}^{m n}(t)=\prec \widetilde{P}_{x y}^{m n}(t) \succ .
$$

ТЕОРемА. Введем обозначения (см. (2), условия 1 и 2)

$$
\eta=\prec \frac{1}{\beta \alpha} \succ-\frac{1}{\beta \alpha}, \quad G=\left(\prec \frac{\lambda}{\beta \alpha} \succ+A\right)^{-1}, \quad \mu=\min \left(1-r_{2}, 1-\frac{r_{1}+r_{2}}{2}\right) .
$$

Тогда, если выполнены:

1) условия 1-4 в разложении среднего пропагатора

$$
\begin{aligned}
P(\lambda)=\prec \widetilde{P}(\lambda) \succ=\lambda^{-1}\left(1-\prec \alpha A G \alpha^{-1} \succ\right)- \\
\quad-\sum_{s=1}^{S} \lambda^{s-1} \prec \alpha A(G \eta)^{s} G \alpha^{-1} \succ-\lambda^{S} \prec \alpha A(G \eta)^{S+1} \beta \frac{1}{\lambda+\alpha A \beta} \succ ;
\end{aligned}
$$

2) условия 1-3 и 5 в разложении

$$
\begin{gathered}
P(\lambda)=\lambda^{-1}\left(1-\prec \beta^{-1} G A \beta \succ\right)-\sum_{s=1}^{S} \lambda^{s-1} \prec \beta^{-1}(G \eta)^{s} G A \beta \succ- \\
-\lambda^{S} \prec \frac{1}{\lambda+\alpha A \beta} \alpha(\eta G)^{S+1} A \beta \succ ;
\end{gathered}
$$

3) условия 1-3 и 6 в разложении

$$
P(\lambda)=\sum_{s=0}^{S} \prec \beta^{-1}(G \lambda \eta)^{s} G \alpha^{-1} \succ+\prec \beta^{-1}(G \lambda \eta)^{S+1} \beta \frac{1}{\lambda+\alpha A \beta} \succ
$$

4) условия 1-3 и 7 в разложении

$$
P(\lambda)=\sum_{s=0}^{S} \prec \beta^{-1}(G \lambda \eta)^{s} G \alpha^{-1} \succ+\prec \frac{1}{\lambda+\alpha A \beta} \alpha(\lambda \eta G)^{S+1} \alpha^{-1} \succ
$$

то текущие члены сумм по s и остатки после $(S+1)$-го члена при $\lambda \rightarrow 0$, $\operatorname{Re}(\lambda) \geqslant 0$, имеют малость вида $O\left(|\lambda|^{s \mu+\epsilon_{1}}\right)$ и соответственно $O\left(|\lambda|^{S \mu+\epsilon_{2}}\right)$. 
ЗАмечАниЕ 1. Четыре пункта теоремы связаны, в частности, с отказом от обязательного сушествования сразу всех величин: $E\left(\beta^{-1}\right), E\left(\alpha^{-1}\right), E(\alpha), E(\beta), E\left(\alpha^{+} \alpha\right)$ или $E\left(\beta \beta^{+}\right)$. Допускается, как и в работах $[3,10,11]$, неограниченное приближение генератора к вырождению в конкретных реализациях случайной среды блужданий.

ЗАМЕЧАНИЕ 2. При одновременном выполнении условий 4-7 различные на первый взгляд представления среднего пропагатора (6)-(9) приводят к одинаковым правильным разложениям при малых $\lambda$.

ДоКАЗАТЕЛЬСТво ТЕОРЕмЫ. Для изучения пунктов 1 и 2 перепишем систему (2) в операторной форме, перейдем к резольвенте и сделаем простейшие преобразования:

$$
\begin{gathered}
\widetilde{P}(\lambda)=\frac{1}{\lambda+\alpha A \beta}, \\
\widetilde{P}(\lambda)=\frac{1}{\lambda}\left(1-\alpha A \frac{1}{\frac{\lambda}{\beta \alpha}+A} \alpha^{-1}\right)=\frac{1}{\lambda}\left(1-\beta^{-1} \frac{1}{\frac{\lambda}{\beta \alpha}+A} A \beta\right), \\
(\widetilde{P}(\lambda))_{x y}^{m n}=\widetilde{P}_{x y}^{m n}(\lambda), \quad 1_{x y}^{m n}=\delta_{x y} \delta_{m n}, \quad(\alpha)_{x y}^{m n}=\alpha_{x}^{m n} \delta_{x y}, \quad(\beta)_{x y}^{m n}=\beta_{x}^{m n} \delta_{x y} .
\end{gathered}
$$

1. Рассмотрим случай, когда выполняется условие $\mathbf{4}$, и возьмем разложение в ряд Неймана (геометрическую прогрессию) с остатком:

$$
\frac{1}{\frac{\lambda}{\beta \alpha}+A}=\sum_{s=0}^{S}(G \lambda \eta)^{s} G+(G \lambda \eta)^{S+1} \frac{1}{\frac{\lambda}{\beta \alpha}+A} .
$$

Подставляя разложение $(12)$ в первое из представлений $\widetilde{P}(\lambda)$ из $(11)$, проведем более подробный анализ усредненных членов появляющегося при этом разложения среднего пропагатора (оператора эволюции) (6) и покажем, что при $\lambda \rightarrow 0, \operatorname{Re}(\lambda) \geqslant 0$, порядки малости текущих членов и остатка этого разложения убывают достаточно быстро.

Для этого член суммы из разложения (6) с индексом $s \geqslant 1$ представим в виде

$$
\begin{aligned}
\lambda^{s-1} \prec & \alpha A(G \eta)^{s} G \alpha^{-1} \succ_{x y}=\sum_{z_{1} \ldots z_{s}} \lambda^{s-1} \prec \alpha_{x} A G_{x z_{1}} \eta_{z_{1}} G_{z_{1} z_{2}} \eta_{z_{2}} \ldots \eta_{z_{s}} G_{z_{s} y} \alpha_{y}{ }^{-1} \succ= \\
= & \sum_{z_{1} \ldots z_{s}} \lambda^{s-1} \prec \alpha_{x-y}(A G)_{x z_{1}} \eta_{z_{1}} G_{z_{1} z_{2}} \eta_{z_{2}} \ldots \eta_{z_{s}} G_{z_{s} y} \alpha_{0}{ }^{-1} \succ= \\
= & \lambda^{s-1}(2 \pi)^{-d} \int_{-\pi}^{\pi} d^{d} k e^{i k(x-y)} \times \\
& \times \sum_{z_{0} \ldots z_{s}} e^{i k z_{0}} \prec \alpha_{z_{0}}(A G)_{z_{0} z_{1}} \eta_{z_{1}} G_{z_{1} z_{2}} \eta_{z_{2}} \ldots \eta_{z_{s}} G_{z_{s} 0} \alpha_{0}{ }^{-1} \succ= \\
= & \lambda^{s-1} \int_{-\pi}^{\pi} \frac{d^{d} k e^{i k(x-y)}}{(2 \pi)^{d}} \times \\
& \times \sum_{z_{0} \ldots z_{s}} e^{i k z_{0}\left[\prec E\left(\alpha_{z_{0}}\right)(A G)_{z_{0} z_{1}} \eta_{z_{1}} G_{z_{1} z_{2}} \eta_{z_{2}} \ldots \eta_{z_{s}} G_{z_{s} 0} E\left(\alpha_{0}-1\right) \succ-\right.} \\
& -\prec\left(E\left(\alpha_{z_{0}}\right)-\alpha_{z_{0}}\right)(A G)_{z_{0} z_{1}} \eta_{z_{1}} G_{z_{1} z_{2}} \eta_{z_{2}} \ldots \eta_{z_{s}} G_{z_{s} 0} E\left(\alpha_{0}-1\right) \succ- \\
& -\prec E\left(\alpha_{z_{0}}\right)(A G)_{z_{0} z_{1}} \eta_{z_{1}} G_{z_{1} z_{2}} \eta_{z_{2}} \ldots \eta_{z_{s}} G_{z_{s} 0} \eta_{0} \succ+ \\
& \left.+\prec\left(E\left(\alpha_{z_{0}}\right)-\alpha_{z_{0}}\right)(A G)_{z_{0} z_{1}} \eta_{z_{1}} G_{z_{1} z_{2}} \eta_{z_{2}} \ldots \eta_{z_{s}} G_{z_{s} 0} \eta_{0} \succ\right] .
\end{aligned}
$$


Приведем анализ последней суммы в (13); остальные суммы в (13) исследуются сходным образом. Важнейшим в анализе этих сумм является учет совпадений индексов суммирования $z_{0}, z_{1}, \ldots$.

Ненулевой вклад в сумму вносят лишь те слагаемые, в которых каждое значение, принимаемое индексами $z_{0}, \ldots, z_{s}$, повторяется хотя бы два раза. Учет этого обстоятельства позволяет разбить полную сумму на конечное число сумм, соответствующих разбиениям $R$ множества $\{0, \ldots, s\}$ на не более чем $(s+1) / 2$ классов, содержаших не менее двух элементов каждый. Сумма переписывается в виде

$$
\begin{aligned}
\lambda^{s-1} \sum_{R} & \sum_{z_{0} \ldots z_{s}} \prod_{i \sim j(R)} \delta_{z_{i} z_{j}} \prod_{i \nsim j(R)}\left(1-\delta_{z_{i} z_{j}}\right) \times \\
& \times e^{i k z_{0}} \prec\left(E\left(\alpha_{z_{0}}\right)-\alpha_{z_{0}}\right)(A G)_{z_{0} z_{1}} \eta_{z_{1}} G_{z_{1} z_{2}} \eta_{z_{2}} \ldots \eta_{z_{s}} G_{z_{s} 0} \eta_{0} \succ,
\end{aligned}
$$

где записи $i \sim j(R)$ и $i \neq j(R)$ означают, что $i$ и $j$ принадлежат (соответственно не принадлежат) одному классу разбиения $R$.

Вследствие трансляционной инвариантности функции $z \rightarrow\left(\alpha_{z}, \beta_{z}\right)$ для каждого $R$ член суммы в (14) представляет собою выражение, зависяшее лишь от $z_{0}-z_{1}, z_{1}-z_{2}, \ldots$ $\ldots, z_{s-1}-z_{s}, z_{s}$. Переписав каждый член этой суммы в виде многочлена относительно всевозможных $\delta_{z_{i}} z_{j}$, мы получим конечную сумму по более грубым, чем $R$ (т.е. объединяюшим классы $R$ ), разбиениям. Подставляя затем вместо $G_{z_{i} z_{j}}$ его представление в виде

$$
(2 \pi)^{-d} \int_{-\pi}^{\pi} d^{d} k e^{i k\left(z_{i}-z_{j}\right)} G(k)
$$

(и с учетом того, что $A G=1-\prec \lambda /(\beta \alpha) \succ G$ ), окончательно приходим к верхней оценке представления (14) конечным числом величин вида

$$
\begin{gathered}
C \mid \lambda^{s-1} \int_{-\pi}^{\pi} d^{d} k_{1} \ldots d^{d} k_{m} G\left(k_{1}\right) \ldots G\left(k_{m}\right) G\left(\sum_{j=1}^{m} c_{j}^{1} k_{j}\right) \times \cdots \\
\ldots \times G\left(\sum_{j=1}^{m} c_{j}^{s-m} k_{j}\right) \exp \left(i \sum_{j, l} b_{j}^{l} z_{j} k_{l}\right) \mid
\end{gathered}
$$

где $2 s>2 m \geqslant s+1$ и $c_{j}^{r}, b_{j l}$ - целые числа.

При оценке величин типа

$$
G\left(\sum_{j=1}^{m} c_{j}^{n} k_{j}\right)
$$

(со "сложным" аргументом) с помошью условия 2 и последуюшей оценке интегралов от $G(k)$, основанной на условии $\mathbf{3}$, мы заключаем, что каждая из частичных сумм имеет порядок $O\left(|\lambda|^{s-1-m r_{2}-(s-m) r_{1}}\right)$, обеспечивая с ростом $s$ последовательное увеличение порядка малости по меньшей мере с шагом $|\lambda|^{\mu}$. 
Аналогичное рассуждение играет основную роль в применении к остаточному члену из (6). Для этого предварительно следует воспользоваться неравенством Коши-Шварца:

$$
\begin{aligned}
\left|\prec \alpha A(G \eta)^{S+1} \beta \frac{1}{\lambda+\alpha A \beta} \succ_{x y}^{m n}\right|^{2} \leqslant & \\
\leqslant & \prec \alpha A(G(\lambda) \eta)^{S+1} \beta \beta^{+}\left(\eta^{+} G(\lambda)^{+}\right)^{S+1} A^{+} \alpha^{+} \succ_{x x}^{m m} \times \\
& \times \prec \frac{1}{\bar{\lambda}+\beta^{+} A^{+} \alpha^{+}} \times \frac{1}{\lambda+\alpha A \beta} \succ_{y y}^{n n} .
\end{aligned}
$$

Второй сомножитель в правой части неравенства (16) оценивается согласно условию 4 величиной $O\left(|\lambda|^{-r_{3}}\right)$. К первому сомножителю применяем рассуждения, основанные на учете совпадения индексов, аналогичные тем, которые использовались в оценке последней суммы (13). Это также дает увеличение порядка степенной малости по $\lambda$ в остаточном члене с шагом не менее $O\left(|\lambda|^{\mu}\right)$.

Следовательно, проводя почленные асимптотические разложения при малых $|\lambda|$ в правой части (6), мы получим в конечном числе слагаемых порядок малости любой требуемой степени.

Отметим, что главный порядок асимптотики при малых $|\lambda|$ во многих рассмотренных в этом пункте случаях определяется из первых двух членов разложения среднего пропагатора:

$$
P(\lambda) \approx \prec \alpha\left(\lambda+A \prec \frac{1}{\beta \alpha} \succ^{-1}\right)^{-1} \alpha^{-1} \succ-\prec \alpha \eta G \alpha^{-1} \succ,
$$

что имеет место, например, в случае сходимости к диффузионноподобному процессу.

2. При выполнении условия 5 воспользуемся правой частью формулы (11) и, подставив в нее разложение, двойственное (12), т.е.

$$
\frac{1}{\frac{\lambda}{\beta \alpha}+A}=\sum_{s=0}^{S}(G \lambda \eta)^{s} G+\frac{1}{\frac{\lambda}{\beta \alpha}+A}(\lambda \eta G)^{S+1},
$$

получим (7) - аналог (6) - и аналоги всех остальных результатов. В частности, здесь часто можно ограничиться двучленной формой

$$
P(\lambda) \approx \prec \beta^{-1}\left(\lambda+\prec \frac{1}{\beta \alpha} \succ^{-1} A\right)^{-1} \beta \succ-\prec \beta^{-1} G \eta \beta \succ,
$$

которая достаточна для получения главных асимптотических членов в большинстве интересных систем.

3. Для разбора двух последних случаев воспользуемся более простым преобразованием резольвенты процесса

$$
\widetilde{P}(\lambda)=\frac{1}{\lambda+\alpha A \beta}=\beta^{-1} \frac{1}{\frac{\lambda}{\beta \alpha}+A} \alpha^{-1} .
$$


При выполнении условия 6 подставим в (20) разложение (12), почленное усреднение которого дает нам (8). Оно исследуется аналогично разложениям (6) и (7).

4. Если же выполнено условие 7, подставим в (20) разложение (18), почленно усредним и придем к (9).

В двух последних случаях удовлетворительная для многих моделей точность получается из соотношения вида

$$
P(\lambda) \approx \prec \beta^{-1} G \alpha^{-1} \succ
$$

Теорема доказана.

Для эффективного вывода из полученных результатов разложения среднего пропагатора при $t \rightarrow+\infty$ следует рассмотреть разложения при малых $|\lambda|$ для матричных элементов (см., например, [10]) и затем воспользоваться либо тауберовыми (абелевыми) теоремами для преобразования Лапласа, либо методом постоянной фазы для обратного преобразования Лапласа [14]. Более подробно это рассматривается на примере следующих двух моделей.

3. Рассмотрим два семейства из СБНРС на многомерной решетке с элементарной ячейкой из двух узлов, описываемых уравнениями типа (2).

Первое семейство описывается уравнениями вида

$$
\begin{aligned}
\frac{d}{d t}\left(\begin{array}{cc}
\widetilde{P}_{x y}^{11} & \widetilde{P}_{x y}^{12} \\
\widetilde{P}_{x y}^{21} & \widetilde{P}_{x y}^{22}
\end{array}\right)= & -\sum_{z}\left(\begin{array}{cc}
\sum_{q}\left(a_{q}+b_{q}\right) \delta_{x-z}-a_{x-z} & -b_{x-z} \\
-b_{z-x} & \sum_{q}\left(b_{q}+c_{q}\right) \delta_{x-z}-c_{x-z}
\end{array}\right) \times \\
& \times\left(\begin{array}{cc}
\alpha_{z} & \beta_{z} \\
\beta_{z} & \gamma_{z}
\end{array}\right)\left(\begin{array}{cc}
\widetilde{P}_{z y}^{11} & \widetilde{P}_{z y}^{12} \\
\widetilde{P}_{z y}^{21} & \widetilde{P}_{z y}^{22}
\end{array}\right),
\end{aligned}
$$

где вешественные величины удовлетворяют условиям:

а) $a_{x}=a_{-x}, c_{x}=c_{-x}, a_{z}, b_{z}, c_{z} \geqslant 0, \delta_{x}=\delta_{x 0}$;

б) при вешественных $k$ сходящаяся сумма матриц

$$
A(k)=\sum_{z}\left(\begin{array}{cc}
a_{z}\left(1-e^{-i k z}\right)+b_{z} & -b_{z} e^{-i k z} \\
-b_{-z} e^{-i k z} & c_{z}\left(1-e^{-i k z}\right)+b_{z}
\end{array}\right)
$$

положительно определена всюду, кроме конечного множества значений $k$;

в) $0 \leqslant \beta_{x}$,

$$
\gamma_{x} \geqslant \beta_{x} \sum_{z} \frac{a_{z}+b_{z}}{b_{0}}, \quad \alpha_{x} \geqslant \beta_{x} \sum_{z} \frac{c_{z}+b_{z}}{b_{0}}
$$

г) существуют первый и достаточно много обратных моментов $\prec \zeta^{-r} \succ, r \geqslant-1$, у независимых при разных $z$ и одинаково распределенных в совокупности ограниченных случайных матриц

$$
\zeta_{z}=\left(\begin{array}{cc}
\alpha_{z} & \beta_{z} \\
\beta_{z} & \gamma_{z}
\end{array}\right)
$$


При сделанных предположениях генератор модели $(22) l_{1}$-ограничен и имеет непрерывный фурье-образ $A(k)$. Оценка $(3)$ с показателем $r_{3}=4$ справедлива для всех комплексных значений параметра Лапласа $\lambda$, лежаших вне конечного круга радиуса

$$
\bar{D}=\frac{\|A \zeta\|_{l_{1}}}{2}=\sup _{z, \alpha, \beta, \gamma} \sum_{q}\left[\left(a_{q}+b_{q}\right)\left(\alpha_{z}+\beta_{z}\right)+\left(b_{q}+c_{q}\right)\left(\beta_{z}+\gamma_{z}\right)\right]
$$

с центром в $(-\bar{D}, 0)$, целиком умещающегося в левой полуплоскости (см. [10, приложение]) и не зависяшего от реализации $\alpha_{x}, \beta_{x}, \gamma_{x}$. Если потребовать, чтобы величины $a_{z}$, $b_{z}, c_{z}$ убывали не слишком медленно при $|z| \rightarrow \infty$, например, как $O\left(|z|^{-s}\right)$ с показателем $s>d$, то требования $r_{1}+r_{2}<2$ и $r_{2}<1$ из условий 2 и 3 выполняются с большим запасом. При этом вид диффузионной асимптотики (т.е. если величина $|x-y|$ конечна, то $P_{x y}(t) \sim t^{-d / 2} \exp [-Q(x-y) / t]$, где $Q(z)$ - положительно-определенная квадратичная форма) обеспечивается конечностью сумм - вторых пространственных моментов

$$
\sum_{z} a_{z} z^{2}, \quad \sum_{z} b_{z} z^{2}, \quad \sum_{z} c_{z} z^{2}
$$

Для степенного убывания последнее означает, что $s>d+2$. Необходимую для подробного исследования случая степенного на бесконечности поведения величин $a_{z}, b_{z}, c_{z}$ технику можно найти в [10].

Таким образом, в случае конечных пространственных моментов (24) в главном порядке по $\lambda$ получаем из (17)

$$
\begin{aligned}
P(\lambda) & \approx \prec \frac{1}{\zeta}\left(\prec \frac{\lambda}{\zeta} \succ+A\right)^{-1} \succ= \\
& =\prec \frac{1}{\zeta} \succ^{1 / 2}\left(\lambda+\prec \frac{1}{\zeta} \succ^{-1 / 2} A \prec \frac{1}{\zeta} \succ^{-1 / 2}\right)^{-1} \prec \frac{1}{\zeta} \succ^{-1 / 2} .
\end{aligned}
$$

Это соответствует долговременной диффузионной асимптотике, которую можно найти с помошью метода Лапласа [14] из асимптотического разложения по большому параметру $t$ интеграла вида

$$
\begin{aligned}
P_{x y}(t) \approx & \prec \frac{1}{\zeta} \succ^{1 / 2} \int_{-\pi}^{\pi} \frac{d^{d} k}{(2 \pi)^{-d}} \times \\
& \times \exp \left[i k(x-y)-t \prec \frac{1}{\zeta} \succ^{-1 / 2} A(k) \prec \frac{1}{\zeta} \succ^{-1 / 2}\right] \prec \frac{1}{\zeta} \succ^{-1 / 2} .
\end{aligned}
$$

Предложенная система отличается от известных многомерных моделей тем, что в ней на два узла приходятся три локальных случайных параметра. Совершенно аналогичным образом с помощью $(N \times N)$-матриц строятся асимптотически точно решаемые модели СБНРС, в которых на $N$ узлов элементарной ячейки приходятся $N(N+1) / 2$ независимых локальных параметров. Если же не ограничиваться случаем рассмотренного для 
простоты эрмитова оператора $G$ (см. теорему) с эрмитовыми $A$ и $\zeta$, число параметров на узел может достигать $N$.

4. Второе, симметричное семейство асимптотически точно решаемых моделей имеет вид

$$
\begin{aligned}
\frac{d}{d t}\left(\begin{array}{cc}
\widetilde{P}_{x y}^{11} & \widetilde{P}_{x y}^{12} \\
\widetilde{P}_{x y}^{21} & \widetilde{P}_{x y}^{22}
\end{array}\right)= & -\sum_{z}\left(\begin{array}{cc}
\alpha_{x} & 1-\beta_{x} \\
1-\alpha_{x} & \beta_{x}
\end{array}\right)\left(\begin{array}{cc}
a_{x-z} & b_{x-z} \\
b_{z-x} & c_{x-z}
\end{array}\right) \times \\
& \times\left(\begin{array}{cc}
\alpha_{z} & 1-\alpha_{z} \\
1-\beta_{z} & \beta_{z}
\end{array}\right)\left(\begin{array}{cc}
\widetilde{P}_{z y}^{11} & \widetilde{P}_{z y}^{12} \\
\widetilde{P}_{z y}^{21} & \widetilde{P}_{z y}^{22}
\end{array}\right)
\end{aligned}
$$

где вешественные величины удовлетворяют следующим условиям:

а) при $x \neq 0$ имеем $a_{x}, b_{x}, c_{x} \leqslant 0$, а также $\sum_{r}\left(a_{r}+b_{r}\right)=0, \sum_{r}\left(c_{r}+b_{r}\right)=0, a_{0}, c_{0} \leqslant 0$ (это обеспечивает такой же вид оператора $A$, как и в случае моделей $(22)$ );

б) при вешественных $k$ сходящаяся сумма матриц

$$
A(k)=-\sum_{z} e^{-i k z}\left(\begin{array}{cc}
a_{z} & b_{z} \\
b_{-z} & c_{z}
\end{array}\right)
$$

положительно определена всюду, кроме конечного множества значений $k$;

в) $\alpha, \beta \in[0,1]$ и $\alpha_{x}\left(1-\alpha_{x}\right) a_{0}+\left[\left(1-\alpha_{x}\right)\left(1-\beta_{x}\right)+\alpha_{x} \beta_{x}\right] b_{0}+\beta_{x}\left(1-\beta_{x}\right) c_{0} \leqslant 0$;

г) у не зависимых при разных $x$ и одинаково распределенных матриц

$$
\zeta_{x}=\left(\begin{array}{cc}
\alpha_{x} & 1-\beta_{x} \\
1-\alpha_{x} & \beta_{x}
\end{array}\right)
$$

имеется момент $E\left(\zeta^{-1}\right)$ и достаточно много обратных моментов вида $\prec\left(\zeta_{x}^{+} \zeta_{x}\right)^{-n} \succ$, $n>0$.

Проверка условий $\mathbf{2}$ и $\mathbf{3}$ и какого-либо из условий 4-7 на резольвенту здесь столь же проста, как и в предшествуюшем примере, и опирается на очевидные $l_{1}$-ограниченность $A$ и непрерывность $A(k)$, а также на ограниченность матриц $\gamma$. В частности, оценка (3) с показателем $r_{3}=4$ справедлива для всех комплексных значений параметра Лапласа $\lambda$, лежащих вне конечного круга радиуса

$$
\bar{D}=\frac{1}{2} \sup _{\zeta}\left\|\zeta A \zeta^{+}\right\|_{l_{1}}
$$

с центром в $(-\bar{D}, 0)\left(\bar{D}\right.$ - половина $l_{1}$-нормы генератора $\left.(26)\right)$, целиком умешаюшегося в левой полуплоскости и не зависяшего от реализации случайной среды $[10$, приложение].

При конечных пространственных моментах (24) в главном порядке по $\lambda$ получаем из (21)

$$
\begin{aligned}
P(\lambda) \approx & \prec \frac{1}{\zeta^{+}}\left(\prec \frac{\lambda}{\zeta^{+} \zeta} \succ+A\right)^{-1} \frac{1}{\zeta} \succ= \\
= & \prec \frac{1}{\zeta^{+}} \prec \frac{1}{\zeta^{+} \zeta} \succ^{-1 / 2}\left(\lambda+\prec \frac{1}{\zeta^{+} \zeta} \succ^{-1 / 2} A \prec \frac{1}{\zeta^{+} \zeta} \succ^{-1 / 2}\right)^{-1} \times \\
& \times \prec \frac{1}{\zeta^{+} \zeta} \succ^{-1 / 2} \frac{1}{\zeta} \succ
\end{aligned}
$$


что соответствует долговременной диффузионной асимптотике, получаемой методом Лапласа [14] из асимптотического разложения выражения

$$
\begin{gathered}
P_{x y}(t) \approx \prec \frac{1}{\zeta^{+}} \breve{\zeta} \int_{-\pi}^{\pi} \frac{d^{d} k}{(2 \pi)^{-d}} \exp [i k(x-y)-t \breve{\zeta} A(k) \breve{\zeta}] \breve{\zeta} \frac{1}{\zeta} \succ, \\
\breve{\zeta}=\prec \frac{1}{\zeta^{+} \zeta} \succ^{-1 / 2} .
\end{gathered}
$$

Необходимую для подробного исследования и асимптотического разложения пропагатора технику (в важном случае степенного на бесконечности поведения величин $a_{z}, b_{z}, c_{z}$ ) можно найти в [10].

Сходным образом для всякого натурального $N$ строятся системы с элементарной ячейкой из $N$ узлов и симметричным вперед-назад переносом, в которых на каждый узел приходится по $(N-1)$ независимых случайных переменных. С этой целью вместо использованной двухрядной матрицы $\zeta$ следует взять случайную $(N \times N)$-матрицу с единичной суммой элементов в каждом столбце.

5. Прямое обобшение описанного выше метода асимптотического исследования СБНРС позволяет рассматривать следуюшие более обширные классы моделей вида (2), где на случайные параметры накладываются менее строгие ограничения, допускаюшие вместо независимости быстрое убывание корреляций:

a) распределение решеточной функшии (принимаюшей в качестве значений пары $(N \times N)$-матриц) $z \rightarrow\left(\alpha_{z}, \beta_{z}\right)$ инвариантно к сдвигам решетки;

б) при некотором конечном $R_{0}$ из $|x-y| \geqslant R_{0}$ следует независимость пар $\left(\alpha_{x}, \beta_{x}\right)$ и $\left(\alpha_{y}, \beta_{y}\right)$.

В этих предположениях доказательства пригодности какого-нибудь из выражений (6)-(9) для получения асимптотического разложения пропагатора отличаются лишь тем, что в выражениях типа (13) отличными от нуля могут быть только слагаемые, в которых для каждого $z_{i}$ найдется еще хотя бы один $z_{j}$, отстоящий от первого менее чем на $R_{0}$.

6. Значительно более разнообразны асимптотически точно решаемые модели, в которых вместо матричнозначных функций $z \rightarrow\left(\alpha_{z}, \beta_{z}\right)$ (см. (2)) фигурируют операторнозначные решеточные функции

$$
\frac{d \widetilde{P}_{x y}^{m n}}{d t}=-\sum_{v, w, z} \sum_{i, j, l=1}^{N} \alpha_{x v}^{m i} A_{v-w}^{i j} \beta_{w z}^{j l} \widetilde{P}_{z y}^{l n}, \quad \widetilde{P}_{x y}^{m n}(t=0)=\delta_{x y} \delta_{m n} .
$$

При этом требуется, чтобы независимые (или коррелированные лишь на конечном расстоянии) трансляционно-инвариантно распределенные случайные операторы $\alpha$ и $\beta$ в уравнении (30) имели обратные операторы типа

$$
\left((\beta \alpha)^{-1}\right)_{x y}^{m n}=\sum_{j=1}^{J} \gamma_{x}^{m n \mid j} \delta_{x-y-a_{j}} \gamma_{y}^{m n \mid j+J}
$$


где суммы по $j$ конечны, и решеточные мультиматричные функции $x \rightarrow\left(\gamma_{x}^{\mid 1}, \ldots, \gamma_{x}^{\mid 2 J}\right)$ были случайны.

Таковы, например, семейства операторов $\alpha$ и $\beta$, которые сами представляются выражениями того же вида, которые стоят в правой части (31), с верхними треугольными матрицами на месте $\gamma$. Допустимы и случаи, когда все эти матрицы треугольны снизу либо матрицы для операторов $\alpha$ имеют один “тип треугольности" (верхний либо нижний), а для $\beta$ - другой. Очевидно также утверждение: если каждая из двух операторных функций $(x, y) \rightarrow\left(\alpha_{1 x y}^{-1}, \beta_{1 x y}^{-1}\right)$ и $(x, y) \rightarrow\left(\alpha_{2 x y}^{-1}, \beta_{2 x y}^{-1}\right)$ имеет представление $(31)$, то функции $(x, y) \rightarrow\left(\alpha_{1 x y}^{-1} \alpha_{2 x y}^{-1}, \beta_{1 x y}^{-1} \beta_{2 x y}^{-1}\right)$ и $(x, y) \rightarrow\left(\alpha_{1 x y}^{-1} \alpha_{2 x y}^{-1}, \beta_{2 x y}^{-1} \beta_{1 x y}^{-1}\right)$ также имеют такое представление.

Особенность доказательства основных результатов о поведении элементов асимптотического разложения - аналогов выражений типа (13) - состоит в учете наличия большего числа ненулевых слагаемых в этих выражениях, что не сказывается на сходимости асимптотического разложения. Основные использованные и полученные выше формулы и утверждения превращаются в формулы, отличающиеся лишь большей общностью входящих в них операторов $\alpha$ и $\beta$.

7. Важная область применения указанных асимптотически точно решаемых моделей - проверка используемых в теоретической физике и иных областях "полуматематических" методов.

Предложенные нами асимптотически точно решаемые модели СБНРС допускают точное решение, которое получается недостаточно математически обоснованным методом. Этот метод положен в основу новых способов теоретического анализа и численного моделирования асимптотической стадии реалистических процессов случайных блужданий в неупорядоченных системах [13].

Использование его в системах, которые изучались в данной работе, предполагает рассмотрение периодической случайной среды, которая в применении к уравнениям (30) с данными (31) получается из случайного в некотором кубе с ребром $R$ набора матриц $x \rightarrow\left(\alpha_{x}^{\mid 1}, \ldots, \alpha_{x}^{\mid 2 J} ; \beta_{x}^{\mid 1}, \ldots, \beta_{x}^{\mid 2 J}\right)$ продолжением по $x$ на все пространство с периодом $R$. В такой ситуации фурье-образ (решеточный) генератора процесса при малых величинах нормы вектора параметров Фурье допускает эффективный расчет главного собственного значения по теории возмушений. Получаемый аналитически на этом пути ответ в пределе больших $R$ находится в полном согласии с найденными в настояшей работе точными результатами.

Благодарности. Автор очень признателен Ф. С. Джепарову и Р. А. Минлосу за внимание к работе и ценные замечания.

Работа выполнена при поддержке Российского фонда фундаментальных исследований (проекты 97-01-000714, 96-15-96418, 96-02-17771-а).

\section{Список литературы}

[1] J. Bernasconi, S. Alexander, R. Orbach. Phys. Rev. Lett. 1978. V. 41. P. 185.

[2] Я. Г. Синай. Теория вероятн. и ее примен. 1982. Т. 27. С. 247. 
[3] P. J.H. Denteneer, M. H. Ernst. Phys. Rev. 1984. V. 29. P. 1755.

[4] J.-P. Bouchaud, A. George. Phys. Rep. 1990. V. 185. P. 127.

[5] Ю.Г. Абов, М. И. Булгаков, С. П. Боровлев и др. ЖЭТФ. 1991. Т. 99. С. 962.

[6] Ф. С. Джепаров. ЖЭТФ. 1991. Т. 99. С. 982.

[7] J. Bricmont, A. Kuppiainen. Phys. Rev. Lett. 1991. V. 66. P. 1689; Commun. Math. Phys. 1991. V. 142. P. 345.

[8] J. W. Haus, K. W. Kehr. Phys. Rev. B. 1991. V. 44. P. 4341.

[9] Proc. of 4th Int. Conf. on Hopping and Related Phenomena. Phil. Mag. B. 1992. V. 65. № 4.

[10] Ф. С. Джепаров, В. E. IШестопал. ТМФ. 1993. Т. 94. С. 496.

[11] Ф. С. Джепаров, В. Е. Шестопал. Письма в ЖЭТФ. 1994. Т. 60. С. 178.

[12] M. O. Cáceres, H. Matsuda, T. Odagaki, D. P. Prato, W. Lamberti. Phys. Rev. B. 1997. V. 56. P. 5897.

[13] Ф. С. Джепаров, Д. В. Львов, К.Н. Нечаев, В. Е. Шестопал. Письма в ЖЭТФ. 1995. T. 62. C. 639 .

[14] М. Ф. Федорюк. Асимптотика: интегралы и ряды. М.: Наука, 1987.

Поступила в редакцию 26.VIII.1998 г. 